\title{
Optimal bundle pricing for homogeneous items
}

Citation for published version (APA):

van Loon, J., Grigoriev, A., Uetz, M. J., \& Vredeveld, T. (2006). Optimal bundle pricing for homogeneous items. METEOR, Maastricht University School of Business and Economics. METEOR Research Memorandum No. 050 https://doi.org/10.26481/umamet.2006050

Document status and date:

Published: 01/01/2006

DOI:

10.26481/umamet.2006050

Document Version:

Publisher's PDF, also known as Version of record

\section{Please check the document version of this publication:}

- A submitted manuscript is the version of the article upon submission and before peer-review. There can be important differences between the submitted version and the official published version of record.

People interested in the research are advised to contact the author for the final version of the publication, or visit the DOI to the publisher's website.

- The final author version and the galley proof are versions of the publication after peer review.

- The final published version features the final layout of the paper including the volume, issue and page numbers.

Link to publication

\footnotetext{
General rights rights.

- You may freely distribute the URL identifying the publication in the public portal. please follow below link for the End User Agreement:

www.umlib.nl/taverne-license

Take down policy

If you believe that this document breaches copyright please contact us at:

repository@maastrichtuniversity.nl

providing details and we will investigate your claim.
}

Copyright and moral rights for the publications made accessible in the public portal are retained by the authors and/or other copyright owners and it is a condition of accessing publications that users recognise and abide by the legal requirements associated with these

- Users may download and print one copy of any publication from the public portal for the purpose of private study or research.

- You may not further distribute the material or use it for any profit-making activity or commercial gain

If the publication is distributed under the terms of Article $25 \mathrm{fa}$ of the Dutch Copyright Act, indicated by the "Taverne" license above, 
Alexander Grigoriev, Joyce van Loon, Marc Uetz, Tjark Vredeveld

Optimal Bundle Pricing for Homogeneous Items

$\mathrm{RM} / 06 / 050$

JEL code : C44, C61

\section{METEबrR}

Maastricht research school of Economics of TEchnology and ORganizations

Universiteit Maastricht

Faculty of Economics and Business Administration P.O. Box 616

NL - 6200 MD Maastricht

phone : ++31433883830

fax : ++31433884873 



\title{
Optimal Bundle Pricing for Homogeneous Items
}

\author{
Alexander Grigoriev, Joyce van Loon, Marc Uetz, and Tjark Vredeveld \\ Maastricht University, Quantitative Economics, \\ P.O.Box 616, NL-6200 MD Maastricht, The Netherlands \\ \{a.grigoriev, j.vanloon,m.uetz,t.vredeveld\}@ke.unimaas.nl
}

\begin{abstract}
We consider a revenue maximization problem where we are selling a set of $m$ items, each of which available in a certain quantity (possibly unlimited) to a set of $n$ bidders. Bidders are single minded, that is, each bidder requests exactly one subset, or bundle of items. Each bidder has a valuation for the requested bundle that we assume to be known to the seller. The task is to find an envy-free pricing such as to maximize the revenue of the seller. We derive several complexity results and algorithms for several variants of this pricing problem. In fact, the settings that we consider address problems where the different items are 'homogeneous' in some sense. First, we introduce the notion of affine price functions that can be used to model situations much more general than the usual combinatorial pricing model that is mostly addressed in the literature. We derive fixed-parameter polynomial time algorithms as well as inapproximability results. Second, we consider the special case of combinatorial pricing, and introduce a monotonicity constraint that can also be seen as 'global' envy-freeness condition. We show that the problem remains strongly NP-hard, and we derive a PTAS - thus breaking the inapproximability barrier known for the general case. As a special case, we finally address the notorious highway pricing problem under the global envy-freeness condition.
\end{abstract}

Keywords: Algorithm design, computational complexity, approximation algorithms, price optimization

\section{Introduction}

Consider the situation that we want to sell a set of items to a set of bidders, each of which is interested in exactly one subset, or bundle, of items. We have a certain amount of copies of each item available, and this amount may be limited or unlimited, as in the case of non-digital or digital goods, for example. We assume that each bidder's valuation is known to the seller. A bidder's valuation determines the maximum amount that she is willing to pay for her bundle. We need to determine two things, namely which of the bidders receive their requested bundles, and how much each of them needs to pay. The goal is to maximize the total revenue received from the bidders. A general economic constraint on the possible prices, adopted in this paper as well, is that of envy-freeness. It requires that no bidder is left envious in the sense that she could afford her bundle, but doesn't receive it ${ }^{1}$. This is the general setting for the pricing problems studied in this paper.

In a sequence of recent papers $[1,2,4,5,8-10]$, several algorithms and complexity results have been derived for such price optimization problems. The pricing model that is assumed in all these papers is combinatorial pricing, where each item is assigned an (anonymous) price, and bundle prices are defined by the sum of the respective item prices. We contribute to this line of research in two different directions.

First, we propose a model for determining bundle prices that generalizes the usual combinatorial pricing model. In the model we propose, the price of the bundles is determined

\footnotetext{
${ }^{1}$ More generally, envy-freeness requires that in an allocation, the bundle allocated to a bidder belongs to her demand set, which is the set of all allocations that maximize the bidder's utility [12].
} 
on the basis of affine functions that (may) differ from bidder to bidder, defined on a joint set of variables. Let us give an illustrating example: Each bidder has an individual fixed cost when purchasing the bundle. The seller needs to determine a per-item price, identical for all items. The price paid by any bidder that gets assigned her bundle is determined by her fixed cost, augmented by the cost for all items in her bundle. The goal is to determine the per-item price such as to maximize total revenue. In this case, the price paid by any customer is an affine function that depends on the size of the requested bundle. Clearly, this pricing model implicitly assumes that all items are indeed 'homogeneous' in some sense. In general, the affine pricing model allows for many more pricing scenarios, and we can even show that the usual combinatorial pricing model is contained as a special case.

Second, we consider the combinatorial pricing model. Inspired by so-called price ladder constraints that have been proposed in the literature $[1,7]$, we introduce a monotonicity condition that allows us to derive results that break the semi-logarithmic inapproximability barrier known for the general case [5]. This monotonicity condition has the following motivation: Given that the items for sale are homogeneous in the sense that the item prices do not differ 'too much', we impose the condition that the price of any bundle of size $k$ must not exceed the price of a bundle of size $k+1$ or larger, for any $k$. As an example, in the highway pricing problem as considered in [9], this monotonicity condition requires that the price for a shorter journey cannot exceed that of a longer one. In that sense, we can indeed interpret the condition as a generalized, or 'global' envy-freeness condition, contrasted to traditional envy-freeness where a bidder's valuation restricts only the price of the requested bundle, but not the price of other bundles.

In summary, the pricing problems that we consider are best described by the fact that all items are indeed homogeneous in some sense, e.g., they do not differ too much in terms of possible prices. Before we specifically discuss related work and our contribution, let us define the pricing settings more formally.

\section{$1.1 \quad$ Model}

Let $I=\{1, \ldots, m\}$ denote the set of items for sale, and let $J=\{1, \ldots, n\}$ denote the set of bidders. Each bidder $j \in J$ is interested in exactly one subset of items $I_{j} \subseteq I$. In line with notation in auction literature, we call the set $I_{j}$ also the bundle of bidder $j$. The fact that each bidder is only interested in one particular bundle is referred to as single mindedness [11]. Every bidder has a positive valuation $b_{j}$, which is the maximum amount she is willing to pay for the bundle $I_{j}$. We assume that the valuations are at least one, $b_{j} \geq 1, j \in J$, and that they are known to the seller. Let $c_{i}$ denote the number of (identical) copies that are available of item $i \in I$. We consider both the case of unlimited availability of items (that is, $c_{i} \geq n$ for all items $i \in I$ ), and the case of limited availability of items.

A bidder is a winner if she gets assigned her bundle (at an affordable price), and a loser otherwise. The set of winners is denoted by $W \subseteq J$. A solution to the problem is a price $p(j)$ that bidder $j$ has to pay for her bundle $I_{j}$, for all bidders $j \in J$. Later we will be more specific about further restrictions on the prices. A solution is called feasible if all winners can afford their respective bundles, and if no item is oversold. In the setting with single minded bidders considered here, a solution is envy-free if in addition, for all losers the respective bundle is priced higher than their valuation. Let us summarize the above discussion in a definition for the generic pricing problem that we address in the paper.

Definition 1. A feasible and envy-free solution to a pricing problem consists of prices $p(j)$ for all bidders $j \in J$, and a set of winners $W \subseteq J$ that get assigned their bundles such that 
1. every winner $j$ can afford her bundle, that is $p(j) \leq b_{j}$,

2. every loser $j$ cannot afford her bundle, that is $p(j)>b_{j}$,

3. no item is oversold, that is $\sum_{j \in W}\left|\{i\} \cap I_{j}\right| \leq c_{i}$ for all items $i \in I$.

The objective is to find a solution that maximizes the total revenue of the seller, that is, we want to maximize $\sum_{j \in W} p(j)$.

We consider two different models for the computation of prices. In the first (and in fact, more general) model, the price of bidder $j$ 's bundle is determined by an affine function in some dimension $K$ as follows.

$$
p(j)=a_{j 0}+a_{j 1} x_{1}+\cdots+a_{j K} x_{K}, \quad j \in J .
$$

Here, the coefficients $a_{j k}, k=0, \ldots, K$, are arbitrary coefficients that are given for all bidders $j \in J$. These coefficients may, in general, depend on both the bundle $I_{j}$ and bidder $j$ itself. Thus it may be the case that two bidders with the same bundle pay different prices. The pricing problem consists of determining values for the variables $x_{k}, k=1, \ldots, K$. To stress the generality of this definition, let us give two specific examples. If we let $K=1$ and define $a_{j 1}=\left|I_{j}\right|$ for all bidders $j$, the bundle prices are determined by affine functions that depend only on the size of the bundles. (This is the model discussed also earlier.) The optimization problem is to determine the per-item price $x_{1}$. The second example, given in the proof of Theorem 2, shows that the well known combinatorial pricing model is included as a special case. Moreover, the generic formulation in (1) allows to model many more pricing scenarios. For instance, in the above example one may want to incorporate additional components such as fixed costs per bidder $j$ (letting $a_{j 0} \neq 0$ ) or quantity discounts (e.g., letting $K=2$ and $\left.a_{j 2}=-\log \left|I_{j}\right|, j \in J\right)$.

The second pricing model that we consider is the usual combinatorial pricing, where we have to determine item prices $p_{i}$ for all items $i \in I$, and the price of any bundle $I^{\prime} \subseteq I$ is just the sum of the respective item prices, $p\left(I^{\prime}\right)=\sum_{i \in I^{\prime}} p_{i}$. Given that several inapproximability results exist for this model $[5,8,9]$, we introduce a monotonicity restriction on the set of item prices which we call global envy-freeness. Specifically, we impose that the following holds true for any two subsets of items $I^{\prime}$ and $I^{\prime \prime}$.

$$
p\left(I^{\prime}\right) \leq p\left(I^{\prime \prime}\right) \text { whenever }\left|I^{\prime}\right|<\left|I^{\prime \prime}\right| .
$$

In fact, this is a strong condition, as it imposes that the items -although different- are homogeneous in a certain sense. More specifically, the condition yields that item prices are of the same order of magnitude for all items. However, the condition has a meaningful economic interpretation in a lot of settings where items are of the same type, as it only requires that larger bundles are at least as expensive as smaller ones. We show how global envy-freeness can be exploited to derive results that break the inapproximability barrier known for the general (unconstrained) case.

In the remainder of this paper, we denote by a $\rho$-approximation algorithm, an algorithm that produces a solution with value at least $1 / \rho$ times the optimal solution value. We will call $\rho$ the approximation factor, or guarantee, of the algorithm. Note that this definition ensures that $\rho \geq 1$.

\subsection{Related work}

The problem that is mainly addressed in the literature is the one with unlimited availability of items, combinatorial pricing, and the requirement that the solution is envy-free $[1,2,4$, 
$5,9,10]$. For this problem the maximum revenue is hard to approximate to within a semilogarithmic factor in the number of bidders $n$ [5]. In particular, it is unlikely that a constant approximation algorithm exists. For the same problem, Hartline and Koltun [10] present an approximation scheme with almost linear running time, given that the number of distinct items $n$ is constant. Moreover, Balcan and Blum [2] derive an $\mathrm{O}(k)$-approximation, given that each bidder is interested in bundles of at most $k$ items. Finally, there exist two fully polynomial time approximation schemes $[2,4]$ for the problem where the bidders bundles are nested, that is, for any two bundles $I_{j}$ and $I_{j^{\prime}}$ it holds that $I_{j} \subseteq I_{j^{\prime}}, I_{j^{\prime}} \subseteq I_{j}$ or $I_{j} \cap I_{j^{\prime}}=\emptyset$.

Notice that an instance with nested bundles can always be interpreted as a problem with a linear order on the items, and the bundles consist of consecutive items in that linear order. The latter problem, but now without the requirement that bundles are nested, is known as the "highway (pricing) problem" [9]. Even this seemingly simple problem is NPcomplete $[3,4]$. The highway problem is motivated by the problem to determine tolls to be charged for usage of parts of a highway. For that problem, there exists an $\mathrm{O}(\log m)$ approximation [2], and given that the largest valuation is bounded by a constant, also a polynomial time dynamic programming algorithm [9].

For the highway problem where the availability of any item is constantly bounded, and assuming that the solution does not need to be envy-free, there exists an FPTAS [8]. For arbitrary bundles, however, limited availability of items without the requirement of envyfreeness makes the revenue maximization problem inapproximable to within a factor $n^{1-\varepsilon}$, unless $\mathrm{P}=\mathrm{NP}[8]$. The reason is that the resulting winner selection problem allows to encode the independent set problem.

Pricing problems with combinatorial pricing have also been considered in settings where bidders are interested in bundles of size one only, but with different valuations for different items. In other words, bidders are not single minded. In that setting, a price ladder constraint was introduced by Rusmevichientong et al. [7]. The price ladder says that the relative ordering of prices of the items is known in advance. They leave open the computational complexity of the problem, and provide a heuristic together with encouraging computational results. This price ladder condition is also considered by Aggarwal et al. [1]. They derive results on the computational complexity, as well as approximation algorithms for several variants of the problem. Our definition of global envy-freeness is in fact inspired by these papers.

\subsection{Our results}

For the revenue maximization problem where bundle prices are determined via arbitrary affine price functions, we propose an algorithm with a time complexity $\mathrm{O}\left(\left(K^{3}+n K\right)(n+\right.$ $K)^{K}$ ) time. Here, parameter $K$ is the dimension in which the affine price functions live. In particular, for $K=1$ this is $\mathrm{O}\left(n^{2}\right)$. This result holds for both limited and unlimited availability of the items.

For the same problem with non-constant $K$, and unlimited availability of the items, the maximum revenue is hard to approximate to within a semi-logarithmic factor in the number of bidders $n$. Specifically, fixing an arbitrarily small $\varepsilon>0$, under the assumption that $N P \nsubseteq B P T I M E\left(2^{n^{\varepsilon}}\right)$, the maximum revenue cannot be approximated to within a factor $\mathrm{O}\left(\log ^{\sigma} n\right)$, for some constant $\sigma$ depending on $\varepsilon$. In fact, this follows directly from the corresponding result by Demaine et al. [5], as combinatorial pricing is a special case of the more general setting with affine price functions. In addition, for the same pricing problem 
with limited availability of items, we prove that it is even NP-complete to approximate the maximum revenue to within a factor of $n^{1-\varepsilon}$ of optimum, where $n$ is the number of bidders.

For the revenue maximization problem with combinatorial pricing, we derive (strong) NP-hardness under the global envy-freeness constraint. Moreover, we derive a PTAS for that problem, with a time complexity of $\left.\mathrm{O}\left(n m^{6 / \varepsilon}(\log B)\right)^{6 / \varepsilon}\right)$, where $B=\max _{j} b_{j}$. This PTAS works for both the problem with limited and unlimited availability of items. Finally, for the highway problem under a relaxed version of the global envy-freeness constraint, we derive a $\mathrm{O}(\log B)$-approximation algorithm.

\section{$2 \quad$ Pricing with affine price functions}

For this section, we assume a revenue maximization problem with bidder prices $p(j)$ that are determined via affine price functions $p(j)=a_{j 0}+a_{j 1} x_{1}+\cdots+a_{j K} x_{K}$, one for each bidder $j \in J$, as in equation (1). We distinguish between unlimited and limited availability of items.

\subsection{Unlimited availability of items}

We describe a simple algorithm that solves this problem in polynomial time, as long as the dimension $K$ of the affine price functions is constant. This algorithm is based on a polyhedral characterization of an optimum solution. To that end, suppose that we know which of the bidders are winners in an optimum solution, say $W \subseteq J$. Then we know that the variables $x_{1}, \ldots, x_{K}$ have to fulfill the $|W|$ inequalities

$$
a_{j 0}+a_{j 1} x_{1}+\cdots+a_{j K} x_{K} \leq b_{j}, \quad j \in W .
$$

Denote by $\mathcal{P}$ the polyhedron defined by these $|W|$ inequalities. For an optimum solution $x=\left(x_{1}, \ldots, x_{K}\right)$, at least one of these inequalities must be tight, because otherwise the same set of winners could be charged even more. Assume that $W^{\prime} \subseteq W$ are the bidders for which the above inequalities are tight, and note that $W^{\prime}$ is nonempty. Then the system

$$
a_{j 0}+a_{j 1} x_{1}+\cdots+a_{j K} x_{K}=b_{j}, \quad j \in W^{\prime}
$$

defines a (nonempty) face $\mathcal{F}$ of the polyhedron $\mathcal{P}$. By definition, any point $x \in \mathcal{F}$ defines an optimal solution. Clearly, at most $K$ inequalities are required to completely characterize the face $\mathcal{F}$. Moreover, we have exactly $\operatorname{dim}(\mathcal{F})$ free variables in the optimal solution $x$. In other words, the same total revenue can be obtained by fixing the $\operatorname{dim}(\mathcal{F})$ free variables among $x_{1}, \ldots, x_{K}$ to 0 . Hence, an optimal solution can be obtained by considering all solutions $x$ that are characterized by exactly $K$ out of the following $n+K$ equalities.

$$
\begin{aligned}
& a_{j 0}+a_{j 1} x_{1}+\cdots+a_{j K} x_{K}=b_{j}, \quad j \in J, \\
& x_{k}=0, \quad k=1, \ldots, K .
\end{aligned}
$$

This insight can be used to define a simple algorithm that solves the revenue maximization problem in polynomial time, as long as $K$ is constant.

Theorem 1. Algorithm 1 solves the revenue maximization problem with affine price functions and unlimited availability of items in $O\left(\left(K^{3}+n K\right)(n+K)^{K}\right)$ time. 


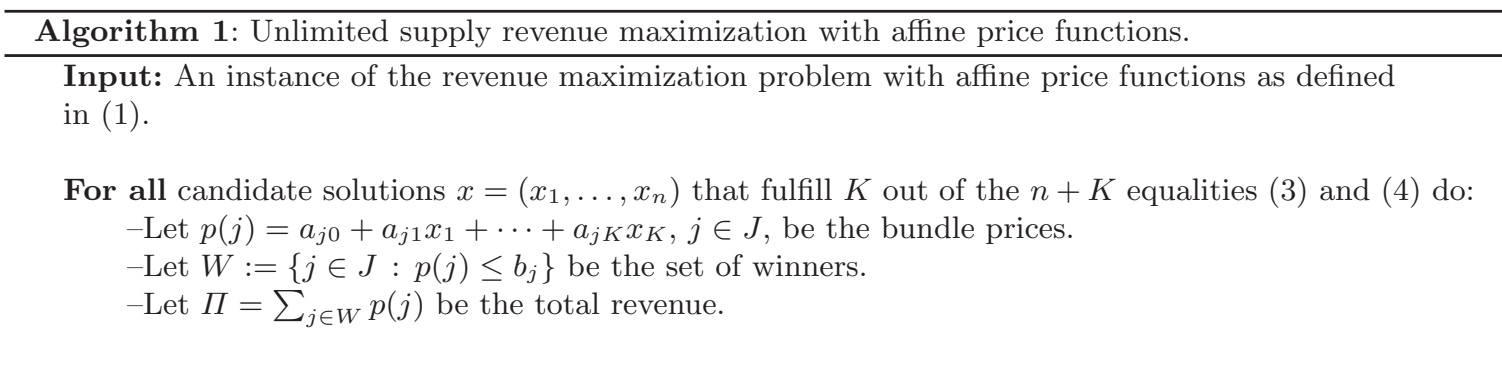

Output: Maximum among all values $\Pi$, with optimal parameters $x_{1}, \ldots, x_{K}$, and set of winners $W$.

Proof. Correctness of the algorithm immediately follows from the preceding discussion. We need to consider $\left(\begin{array}{c}n+K \\ K\end{array}\right) \in \mathrm{O}\left((n+K)^{K}\right)$ systems of $K$ equalities each. In each of these iterations, we need to solve a linear system in $K$ variables and $K$ constraints, which takes $\mathrm{O}\left(K^{3}\right)$ time. Computation of the bundle prices, winners, and the objective value takes $\mathrm{O}(n K)$ time. The claimed time complexity follows.

In contrast, if the dimension $K$ of the price functions is not constant, the optimum revenue is hard to approximate to within a semi-logarithmic factor.

Theorem 2 (see [5]). Fix some small $\varepsilon>0$. Under the assumption that NP $\nsubseteq$ $B P T I M E\left(2^{n^{\varepsilon}}\right)$, the revenue maximization problem with affine price functions and unlimited availability of items is hard to approximate to within a factor $\Omega\left(\log ^{\sigma} n\right)$, for some constant $\sigma$ depending on $\varepsilon$.

Proof. The claim follows from the fact that the problem contains the well known combinatorial pricing model as a special case. Let $K=m$, and for all bidders $j \in J$ and all items $i \in I$, let $a_{j i}=1$ if item $i$ is contained in bundle $I_{j}$, and $a_{j i}=0$ otherwise. Then $x_{i}$ can be interpreted as the price of item $i$, and the price of any bundle $I_{j}$ equals the sum of its item prices, $\sum_{i \in I_{j}} x_{i}$. The optimization problem is to determine optimal item prices. For this problem, the above hardness result was derived by Demaine et al. [5].

\subsection{Limited availability of items}

First we claim that Algorithm 1 can as well be used to solve the problem when the availability of items is limited. Indeed, the only thing we additionally need to check for any of the candidate solutions is feasibility: We have to verify whether none of the items is oversold, and if yes, we do not consider the candidate solution. Clearly, this feasibility check can be done in $\mathrm{O}(\mathrm{nm})$ time per candidate solution.

Corollary 1. Algorithm 1, augmented with a feasibility check, solves the revenue maximization problem with affine price functions and limited availability of items in $\mathrm{O}\left(\left(K^{3}+\right.\right.$ $\left.n K+n m)(n+K)^{K}\right)$ time.

On the negative side, it turns out that the problem with limited availability of the items seems even harder to approximate, as we can show the following.

Theorem 3. Consider the revenue maximization problem with affine price functions and limited availability of items. For any $\varepsilon>0$, it is NP-hard to approximate the maximum revenue to within a factor $n^{1-\varepsilon}$. This result holds even if all bidders have unit valuations, the availability of each item is one, and each item is requested by at most two bidders. 
Proof. We use an approximation preserving reduction from IndePEndetSet. Given is a graph $G=(V, E)$, we want to find a maximum cardinality subset $V^{\prime} \subseteq V$ such that no two vertices in $V^{\prime}$ are adjacent. Zuckerman [13] showed that it is NP-hard to approximate the maximum cardinality independent set to within a factor $|V|^{1-\varepsilon}$, for any $\varepsilon>0$.

We construct the following instance of the pricing problem. Each vertex $v \in V$ corresponds to a bidder and each edge $e \in E$ corresponds to an item. Each bidder $v$ requests a bundle containing all edges incident to $v$, and has valuation $b_{v}=1$. Each item $e$ is available only once $\left(c_{e}=1\right)$. We let the price functions be $p(v)=1+x_{v}$, for all bidders $v \in V$.

We claim that an independent set of cardinality $s$ exists in $G$ if and only if there exists a pricing for the above defined instance with total revenue $s$. Suppose $V^{\prime} \subseteq V$ is an independent set in $G$ with cardinality $s$. Then let $x_{v}=0$ for all $v \in V^{\prime}$, and $x_{v}>0$ otherwise. This way the set of winners equals the independent set $V^{\prime}$, and therefore no item is oversold. No loser is envious, as the bundle price exceeds her valuation, and we extract a total revenue of $s$.

Conversely, assume that we have a solution to the pricing problem with total revenue $s$. Since only one copy of any item is available, the set of winners must define an independent set in $G$. As the maximum revenue from any bidder is 1 , there exists an independent set of size $s$ in $G$.

\section{Combinatorial pricing with global envy-freeness}

In combinatorial pricing, we need to assign an (anonymous) item price for each of the items, and bidder prices $p(j)$ are defined as the sum of the prices of the requested items. To be in line with previous papers on the same topic, from now on let us write $p_{i}$ for the price of item $i$. As before, the item prices need to yield a feasible and envy-free solution, and we wish to maximize the total revenue, which can be written as $\sum_{j \in W} \sum_{i \in I_{j}} p_{i}$. Notice that in case of unlimited availability of items both feasibility and envy-freeness is in fact no issue - yet finding optimal prices is hard by Theorem 2. For this reason we introduce a new condition, namely global envy-freeness, which is inspired by so called price ladder constraints that have been proposed before in slightly different settings $[1,7]$. The condition was already defined in (2), we require that $p\left(I^{\prime}\right) \leq p\left(I^{\prime \prime}\right)$ if $\left|I^{\prime}\right|<\left|I^{\prime \prime}\right|$, for any two subsets of items $I^{\prime}$ and $I^{\prime \prime}$.

\subsection{Complexity}

Theorem 4. Consider the revenue maximization problem with combinatorial pricing and unlimited availability of items. If prices are required to be integral, this problem is NP-hard under the global envy-freeness condition.

Proof. We use a reduction from the NP-complete problem IndePEndetSeT [6]. Let $G=$ $(V, E)$ be a graph in which we want to find a maximum cardinality set of vertices that are pairwise not adjacent. We define an instance for the pricing problem that consists of $|E|$ gadgets, one for each edge $e=\{u, v\}$. Each gadget consists of two vertex-items, $u$ and $v$, and one edge-item $e$. So there are $m=|V|+|E|$ items. For each gadget, we have 7 bidders. For each vertex-item $v \in V$ there is one bidder requesting bundle $\{v\}$, and for each edge-item $e \in E$ there is one bidder requesting bundle $\{e\}$ (we call them singleton bidders). Moreover, there are 4 bidders requesting two-elementary bundles: Two bidders requesting the bundles $\{u, e\}$ and $\{v, e\}$, respectively, and two bidders both requesting the bundle $\{u, v\}$. In total there are thus $n=|V|+5|E|$ bidders. Singleton bidders have valuation $M+1$, and bidders 
requesting a two-elementary bundle have a valuation $2 M+1$, for some $M$ large enough. We claim that there exists an independent set of size $s$ in $G$ if and only if there is a solution for the revenue maximization problem with revenue $M|V|+(9 M+3)|E|+s$.

First, suppose there exists an independent set $V^{\prime}$ in $G$ of size $\left|V^{\prime}\right|=s$. Let $E_{0}=\{e \in$ $\left.E: V^{\prime} \cap e=\emptyset\right\}$ be the set of edges to which no vertex in the independent set is incident. We set the prices of all vertex-items in $V^{\prime}$ and all edge-items in $E_{0}$ to $M+1$, and all other items are priced at $M$. Given that $M$ is large enough, these prices fulfill the global envy-freeness constraint. Next, by definition of $E_{0}$ each bidder can afford her bundle. The total revenue from singleton bidders is $M|V|+s+M|E|+\left|E_{0}\right|$. For each gadget, out of the corresponding three items two are priced at $M$, and one is priced at $M+1$. If the edge-item $e$ is priced $M+1$, the 4 non-singleton bundles of a gadget yield a total revenue $8 M+2$. If one of the two node-items is priced $M+1$, this revenue is $8 M+3$. Thus we have a total revenue $M|V|+s+M|E|+\left|E_{0}\right|+\left|E_{0}\right|(8 M+2)+\left|E \backslash E_{0}\right|(8 M+3)$, which equals $M|V|+(9 M+3)|E|+s$, as claimed.

Conversely, consider the pricing problem. Given that $M$ is large enough, in an optimum solution any item will be priced either $M$ or $M+1$. Indeed, first observe that the price of any item is at most $M+1$. For suppose that there is an item with a price at least $M+2$, the singleton bidder requesting this item cannot afford it, resulting in a total revenue of at most $(M+1)(|V|+|E|-1)+(2 M+1) 4|E|=(M+1)|V|+(9 M+5)|E|-(M+1)<$ $M|V|+(9 M+3)|E|$, for $M \geq|V|+2|E|$. Given that any item price is at most $M+1$, and given the valuations, it is clear that no item will be priced cheaper than $M$ in an optimal solution. In the same way we can argue that each bidder must be a winner in any optimal solution.

We now claim that, in an optimal solution, the set $V^{\prime}=\left\{v \in V: p_{v}=M+1\right\}$ is an independent set in $G$. To see this, observe that for each gadget exactly one of its three items is priced $M+1$. If it was more than one, at least one bidder would be lost. If all items are priced $M$, we could increase the revenue by two, increasing the price of the edge-item by 1 . Moreover, any two vertex-items priced $M+1$ cannot correspond to adjacent vertices in $G$, as two bidders would be lost. Hence, we can write the total revenue of any optimal solution similarly as before, and it equals $M|V|+(9 M+3)|E|+\left|V^{\prime}\right|$. This completes the proof.

The above proof only holds for the problem restricted to integral priced. We can however adapt the instance such that for every vertex- and edge-item there is 1 customer interested in that item and values it at $M$, and there are $M$ customers with valuations $M+1$. In Appendix A, we show why this adaptation gives the proof for non-integral prices. In the next section, we derive a PTAS for the problem that works for both, integral or non-integral pricing.

\subsection{Approximation scheme}

In order to derive a PTAS for the problem with combinatorial pricing and global envyfreeness, we restrict the prices to powers of $(1+\delta)$ for some $\delta>0$. Assume, without loss of generality, that $p_{1} \leq p_{2} \leq \ldots \leq p_{m}$, then by global envy-freeness, we know $2 p_{2} \geq p_{1}+p_{2} \geq$ $p_{m}$. Similarly, $3 p_{3} \geq p_{1}+p_{2}+p_{3} \geq p_{m-1}+p_{m} \geq 2 p_{m-1}$, etc. In general, we know

$$
k p_{k} \geq(k-1) p_{m-k+2}, \quad k=2, \ldots,\left\lceil\frac{m}{2}\right\rceil .
$$

The idea for the PTAS is now the following. Except for a constant number of the cheapest and most expensive items, all items have prices in roughly the same range. Therefore we 
can price all except a constant number of items uniformly with the same price, without loosing too much in terms of the total revenue. We therefore enumerate over all possible uniform prices for the bulk of the items, and over all possible combinations of prices for the remaining (constant number of) items.

Theorem 5. The pricing problem with unlimited availability of items, combinatorial pricing and global envy-freeness admits a PTAS. The time complexity is $O\left(n m^{6 / \varepsilon}(\log B)^{6 / \varepsilon}\right)$, where $\varepsilon$ is the precision of the PTAS and $B=\max _{j} b_{j}$.

Proof. Let $0<\varepsilon<1, \delta=\varepsilon / 3$, and for convenience assume that $1 / \delta$ is integral. Assume that we know the order of prices, say $p_{1} \leq \cdots \leq p_{m}$, in an optimum globally envy-free solution. Define the subsets of items $S=\left\{i \in I: i \leq \frac{1}{\delta}\right\}, M=\left\{i \in I: 1+\frac{1}{\delta} \leq i \leq m+1-\frac{1}{\delta}\right\}$ and $L=\left\{i \in I: i \geq m+2-\frac{1}{\delta}\right\}$. We may assume that $S \cap L=\emptyset$, for otherwise the number of items is constant. We round down the prices of all items in $S$ and $L$ to powers of $(1+\delta)$. Moreover, we price all items in $M$ uniformly at price $p_{1+1 / \delta}$, rounded down to a power of $(1+\delta)$. Let us call the new prices $p^{\prime}$, and let us call $p_{M}^{\prime}$ the price of items in $M$. First observe that the order of prices does not change. Let us next argue that we do not loose too much by this rounding. Clearly, since we only round down, the set of winners can only increase. Moreover, we loose at most a factor $(1+\delta)$ on items in $S$ and $L$. Finally, consider the items in $M$. By (5), we have

$$
\left(1+\frac{1}{\delta}\right) p_{1+1 / \delta} \geq \frac{1}{\delta} p_{m+1-1 / \delta}
$$

In other words, the price for the most expensive item in $M$ differs from the cheapest item in $M$ by a factor at most $(1+\delta)$. Hence, on items in $M$ we loose a factor at most $(1+\delta)^{2}$.

Now we have a structured solution, but it may violate the global envy-freeness condition. We claim that any such violation can be restored by one more rounding operation, if necessary: We just round down the price of all items priced $p_{M}^{\prime}$ or higher by another factor $(1+\delta)$. For contradiction, after this last rounding consider two violating sets $I^{\prime}$ and $I^{\prime \prime}$ with $\left|I^{\prime}\right|<\left|I^{\prime \prime}\right|$ and $p^{\prime}\left(I^{\prime}\right)>p^{\prime}\left(I^{\prime \prime}\right)$, and w.l.o.g. $\left|I^{\prime}\right|=\ell$, and $\left|I^{\prime \prime}\right|=\ell+1$. Due to the ordering of prices, we then also have that $p^{\prime}(\{1, \ldots, \ell+1\})<p^{\prime}(\{m, m-1, \ldots, m-\ell+1\})$. As long as there are items from $M$ in both sets, we redefine $\ell=\ell-1$, and we keep violating global envy-freeness. But now, all items in $\{1, \ldots, \ell\}$ have been rounded down by a factor at most $(1+\delta)$, and all items in $\{m, m-1, \ldots, m-\ell+1\}$ have been rounded down by a factor at least $(1+\delta)$. This contradicts the global envy-freeness of the optimal solution that we started with.

The PTAS now consists of enumerating all possible structured solutions, which is sufficient to obtain a (globally envy-free) solution that differs from the optimal solution by a factor at most $(1+\delta)^{2}<(1+\varepsilon)$. There are $\left(\begin{array}{c}m \\ -1+2 / \delta\end{array}\right)$ possible choices for sets $S$ and $L$. Since all prices are powers of $(1+\delta)$, there $\operatorname{are} \log B$ possible prices. Given that all items in $M$ have the same price, there are at most $(\log B)^{2 / \delta}$ structured solutions. Computation of the revenue for any such solution takes $\mathrm{O}(\mathrm{nm})$ time. This together with $\delta=\varepsilon / 3$ yields the claimed time complexity.

In the above PTAS we enumerate all possible (structured) solutions. Hence we can simultaneously check feasibility and envy-freeness of any such solution with respect to limited availability of items. Thus we immediately get the following corollary. 
Corollary 2. The pricing problem with limited availability of items, combinatorial pricing and global envy-freeness admits a PTAS.

\subsection{Highway problem with global envy-freeness}

The highway problem can be viewed at as pricing edges on a simple path, and the bundles are subpaths. We explore this problem under the assumption that the prices need to satisfy global envy-freeness. However, as every bundle requested by a bidder consists of consecutive items only, we also require the envy-freeness condition (2) to hold only for bundles of consecutive items. In that sense, condition (2) is in fact relaxed in comparison to the more general (non-consecutive) problem. In the previous section, we noticed that the global envyfreeness condition does not allow for much deviation in item prices, see (5). The global envy-freeness condition restricted to bundles of consecutive items, however, allows for large variations in the item prices. Therefore, both the NP-hardness proof and the PTAS of the previous section do not carry over to the highway pricing problem considered here. Example 1 shows that setting the same price for every item can give a bad approximation, which was not the case in the previous section.

Example 1. Let the number of items and customers be given by $m=n=2^{k}-1$ for some integer $k>0$. Let customer $j$ be interested in item $i=j$, that is, $I_{j}=j$ for all $j \in J$. Customer $j$ 's valuation set with the following procedure:

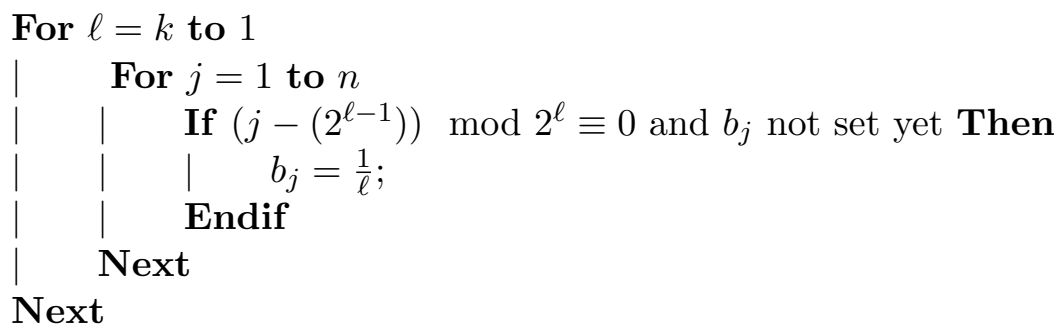

The best uniform pricing is to price all items equal to 1 . This gives a profit of $m=2^{k}-1$. The best optimal price is to let every customer pay up to her valuation, which gives a profit of $\sum_{i=1}^{k} \frac{1}{i} 2^{k-i}$. Thus, the ratio between the optimal profit and the uniform pricing is in $\mathcal{O}(\log \log m)$.

Theorem 6. The highway pricing problem with global envy-freeness is NP-complete.

To prove this theorem, we extend the proof idea for the NP-hardness of the general highway pricing problem in $[3,4]$. We use a reduction from a problem closely related to SuBSETSum. In the transformation to the highway pricing problem, the biggest challenge is to create an instance that allows to decide on the (variant of the) SubsetSum problem, using prices that fulfill the global envy-freeness condition. This is achieved by defining a highway pricing problem with a certain periodic pattern in the bidders' bundles. Details are omitted here; the proof can be found in Appendix B.

We next present a $\left(3+2 \log _{2} B\right)$-approximation algorithm for the highway pricing problem under global envy-freeness, where $B=\max _{j} b_{j}$. To come to this approximation, we present approximation guarantees for two special cases first.

Lemma 1. The highway pricing problem under global envy-freeness in which all bundles have size at least two is approximable within a factor of 3 by optimal uniform pricing. 
Proof. Consider an optimal solution with revenue OPT and let $p_{\max }^{*}$ be the highest item price in this solution. We claim that pricing all items at $p_{\max }^{*} / 3$, yields a revenue of at least OPT $/ 3$. Clearly, an optimal uniform pricing is at least as good as the uniform $p_{\max }^{*} / 3$ pricing.

First, we show that any winner $j \in W$ for the optimal pricing remains a winner for the uniform pricing at level $p_{\max }^{*} / 3$. Let $\left|I_{j}\right|=\ell$. Then the valuation of bidder $j$ is at least $b_{j} \geq\lfloor\ell / 2\rfloor p_{\max }^{*}$, as by the global envy-freeness condition the total price of any two consecutive items in an optimal solution is at least $p_{\max }^{*}$ and client $j$ can afford her bundle. In the uniform $p_{\max }^{*} / 3$ pricing, the total bundle price is $\ell p_{\max }^{*} / 3$, which is at most $\lfloor\ell / 2\rfloor p_{\max }^{*}$, for $\ell \geq 2$. In an optimal pricing client $j$ pays at most $\ell p_{\max }^{*}$, whereas in our uniform pricing, we get $\ell p_{\max }^{*} / 3$. Hence, pricing all items $p_{\max }^{*} / 3$ yields a revenue of at least OPT $/ 3$.

The above lemma shows that whenever all bundles contain at least 2 items, we have a constant approximation. Now, we consider only instances in which bidders request exactly one item. Moreover, we restrict ourselves to instances in which $b_{j} / b_{k} \leq 2$, for any two clients $j$ and $k$.

Property 1. Consider the highway pricing problem under global envy-freeness, restricted to instances in which each bidder requests exactly one item and for each two bidders $j, k$, it holds true that $b_{j} / b_{k} \leq 2$. Pricing each item at $\min _{j} b_{j}$ yields a revenue of at least OPT $/ 2$.

Theorem 7. The best uniform pricing yields a solution with revenue at least $\mathrm{OPT} /(3+$ $\left.2 \log _{2} B\right)$ for the highway pricing problem under global envy-freeness, where $B=\max _{j} b_{j}$. Moreover, the time needed to find this solution is $O\left(n^{2} m\right)$.

Proof. Consider an optimum globally envy-free solution and let $\mathrm{OPT}_{L}$ denote the revenue of bidders with bundle size at least two and let $\mathrm{OPT}_{r}$ denote the revenue of singleton bidders with valuation $2^{r-1} \leq b_{j}<2^{r}\left(r=1, \ldots, \log _{2} B\right)$ in this solution. Then $\mathrm{OPT}=\mathrm{OPT}_{L}+$ $\sum_{r} \mathrm{OPT}_{r}$.

Moreover, let $\mathrm{APP}_{L}$ denote the revenue obtained by the best uniform pricing and $\mathrm{APP}_{r}$ denote the revenue obtained by the best uniform pricing strategy for the bidders in $J_{r}=\{j \in$ $J:\left|I_{j}\right|=1$ and $\left.2^{r-1} \leq b_{j}<2^{r}\right\}$. By Property 1 , we have that $\mathrm{APP}_{r} \geq \mathrm{OPT}_{r} / 2$ and thus $\max _{r} \mathrm{APP}_{r} \geq \sum_{r} \mathrm{OPT}_{r} /\left(2 \log _{2} B\right)$. Moreover, from Lemma 1, it follows that $\mathrm{APP}_{L} \geq \mathrm{OPT}_{L} / 3$. Hence, the solution found yields a revenue of

$$
\max \left\{\mathrm{APP}_{L}, \mathrm{APP}_{r}: r=1, \ldots, \log _{2} B\right\} \geq \mathrm{OPT} /\left(3+2 \log _{2} B\right) .
$$

To see the claim on the time complexity, note that to find the best uniform pricing, we need to consider at most $n$ different prices. For each price, we need to compute the winners and the profit obtained on this price, which can be done in $\mathrm{O}(\mathrm{nm})$ time. So, the best uniform price can be computed in $\mathrm{O}\left(n^{2} m\right)$ time.

\section{Conclusion}

Let us briefly comment on our two NP-hardness proofs for pricing problems that need to satisfy the global envy-freeness condition. Our proof for the NP-hardness of the general combinatorial pricing problem requires prices to be integral; we leave it as an open question if the same problem with non-integral prices is NP-hard as well. In contrast, our proof of NP-hardness of the highway pricing problem does not require prices to be integral. This, however, does not yield NP-hardness of the general combinatorial pricing problem with non-integral prices, as global envy-freeness is more restrictive in the general case. Finally, as global envy-freeness is a strong condition, we believe that a constant factor approximation algorithm exists for the highway pricing problem. 


\section{References}

1. G. Aggarwal, T. Feder, R. Motwani, and A. Zhu, Algorithms for multi-product pricing, Automata, Languages and Programming - ICALP 2004 (J. Díaz, J. Karhumäki, A. Lepistö, and D. Sannella, eds.), Lecture Notes in Computer Science, vol. 3142, Springer, 2004, pp. 72-83.

2. M.F. Balcan and A. Blum, Approximation algorithms and online mechanisms for item pricing, Proc. of the 7th ACM Conference on Electronic Commerce, ACM, 2006, pp. 29-35.

3. H. Bodlaender and E. Penninkx, An elegant NP-completeness proof for profit maximization on paths, personal communication, May 2005.

4. P. Briest and P. Krysta, Single-minded unlimited supply pricing on sparse instances, Proc. 17th Annual ACM-SIAM Symposium on Discrete Algorithms, ACM-SIAM, 2006, pp. 1093-1102.

5. E. D. Demaine, U. Feige, M.T. Hajiaghayi, and M. R. Salavatipour, Combination can be hard: Approximability of the unique coverage problem, Proc. 17th Annual ACM-SIAM Symposium on Discrete Algorithms, ACM-SIAM, 2006, pp. 162-171.

6. M. R. Garey and D. S. Johnson, Computers and intractability: A guide to the theory of np-completeness, W. H. Freeman, New York, 1979.

7. P. W. Glynn, B. Van Roy, and P. Rusmevichientong, A nonparametric approach to multi-product pricing, Operations Research 54 (2006), no. 1, 82-98.

8. A. Grigoriev, J. van Loon, R. Sitters, and M. Uetz, How to sell a graph: Guidelines for graph retailers, Proc. 32nd Int. Workshop on Graph-Theoretic Concepts in Computer Science (F. Fomin, ed.), Lecture Notes in Computer Science, vol. 4271, Springer, 2006, pp. 125-136.

9. V. Guruswami, J. D. Hartline, A. R. Karlin, D. Kempe, C. Kenyon, and F. McSherry, On profitmaximizing envy-free pricing, Proc. 16th Annual ACM-SIAM Symposium on Discrete Algorithms, ACMSIAM, 2005, pp. 1164-1173.

10. J. D. Hartline and V. Koltun, Near-optimal pricing in near-linear time, Algorithms and Data Structures - WADS 2005 (F. K. H. A. Dehne, A. López-Ortiz, and J.-R. Sack, eds.), Lecture Notes in Computer Sciences, vol. 3608, Springer, 2005, pp. 422-431.

11. D. Lehman, L. I. O'Callaghan, and Y. Shoham, Truth revelation in approximately efficient combinatorial auctions, Journal of the ACM 49 (2002), no. 5, 1-26.

12. L. Walras, Elements of pure economics, Allen and Unwin, London, 1954.

13. D. Zuckerman, Linear degree extractors and the inapproximability of MAX CLIQUE and CHROMATIC NUmBer, Electronic Colloquium on Computational Complexity, Report TR05-100 (2005), http://www.eccc.uni-trier.de/eccc/. 


\section{A Proof of Theorem 4 for non-integral prices}

Consider the same construction of the graph as in the proof of Theorem 4, but now with additional customers such that for every vertex- and edge-item there is 1 customer interested in that item and values it at $M$, and there are $M$ customers with valuations $M+1$. We show that for edge $e=\{u, v\}$, pricing vertex-item $u$ at $p_{u}=M+\delta_{u}$, item $v$ at $p_{v}=M+\delta_{v}$ and edge-item $e$ at $p_{e}=M+\delta_{e}$ (where $0<\delta_{u}, \delta_{v}, \delta_{e}<1$ ) will never lead to an optimal solution if $M \geq 3$. First, let $\delta_{u}+\delta_{v}=1$ and $\delta_{u}+\delta_{e}=1$ (similar for $\delta_{v}+\delta_{e}=1$ ). Then, the profit $\Pi_{1}$ is given by

$$
\begin{aligned}
\Pi_{1}= & M\left(M+\delta_{u}\right)+M\left(M+\delta_{v}\right)+M\left(M+\delta_{e}\right)+2\left(M+\delta_{u}+M+\delta_{v}\right) \\
& +\left(M+\delta_{u}+M+\delta_{e}\right)+\left(M+\delta_{v}+M+\delta_{e}\right) \\
\stackrel{\delta_{v}=\delta_{e}}{=1-\delta_{u}} & M^{2}+M \delta_{u}+M^{2}+M\left(1-\delta_{u}\right)+M^{2}+M\left(1-\delta_{u}\right)+6 M+3+2 M+\left(1-\delta_{u}\right)+\left(1-\delta_{u}\right) \\
= & 3 M^{2}-M \delta_{u}+10 M+5-2 \delta_{u} .
\end{aligned}
$$

Now, let $\delta_{e}+\delta_{u}=1$ and $\delta_{e}+\delta_{v}=1$. Then, the profit $\Pi_{2}$ is given by

$$
\begin{aligned}
\Pi_{2}= & M\left(M+\delta_{u}\right)+M\left(M+\delta_{v}\right)+M\left(M+\delta_{e}\right)+\left(M+\delta_{u}+M+\delta_{e}\right) \\
& +\left(M+\delta_{v}+M+\delta_{e}\right)+2\left(M+\delta_{u}+M+\delta_{v}\right) \\
& \stackrel{\delta_{e}=1-\delta_{u}}{=1-\delta_{v}} M^{2}+M \delta_{u}+M^{2}+M \delta_{u}+M^{2}+M\left(1-\delta_{u}\right)+8 M+2\left(1-\delta_{u}\right)+4 \delta_{u} \\
= & 3 M^{2}+M \delta_{u}+9 M+4 \delta_{u}+2 .
\end{aligned}
$$

In the optimal solution, $\delta_{u}=1$ and $\delta_{v}=\delta_{e}=0$. Then, we have

$$
\Pi_{\mathrm{OPT}}=M(M+1)+M+M M+M+M M+3(2 M+1)+2 M=3 M^{2}+11 M+3 .
$$

First we test whether $\Pi_{\mathrm{OPT}} \geq \Pi_{1}$.

$$
3 M^{2}+11 M+3 \geq 3 M^{2}-M \delta_{u}+10 M+5-2 \delta_{u} \Leftrightarrow(M+2) \delta_{u} \geq 2-M \Leftrightarrow \delta_{u} \geq \frac{2-M}{M+2}
$$

As $M \geq 3$, the righthand-side is at most $-1 / 5$ and therefore the inequality holds. Now, let us consider $\Pi_{\mathrm{OPT}} \geq \Pi_{2}$.

$$
3 M^{2}+11 M+3 \geq 3 M^{2}+M \delta_{u}+9 M+4 \delta_{u}+2 \Leftrightarrow 2 M+1 \geq(M+4) \delta_{u} \Leftrightarrow \delta_{u} \leq \frac{2 M+1}{M+4}
$$

For the righthand-side of the last inequality we have

$$
\frac{2 M+1}{M+4}=\frac{M+4}{M+4}+\frac{M-3}{M+4} \stackrel{M \geq 3}{\geq} .
$$

So also this inequality holds, and we can conclude that there exists no solution with fractional prices that is more profitable than $\Pi_{\mathrm{OPT}}$.

\section{B Proof of Theorem 6}

We prove NP-completeness of the highway problem with global envy-freeness by a reduction from EqualCardinalitySubsetSum: Given a set of positive integers $a_{1}, a_{2}, \ldots, a_{2 L}$ and nonnegative integer $A$, does there exist a set $S \subseteq\{1, \ldots, 2 L\}$ such that $\sum_{\ell \in S} a_{\ell}=A$ and $|S|=L$ ? This problem is NP-complete, as shown in Lemma 2. Finally, in Lemma 3 we verify global envy-freeness of the pricing strategy we use in the reduction. 
Lemma 2. EqualCardinalitySubsetSum is NP-complete.

Proof. Given an instance of NP-complete [6] problem SubSETSum. Nonnegative integers $x_{1}, x_{2}, \ldots, x_{n}$ and nonnegative integer $K$, does there exist a set $T \subseteq\{1, \ldots, n\}$ such that $\sum_{i \in T} x_{i}=K$ ? Let $L=n, A=K$ and $a_{\ell}=x_{\ell}$ for all $\ell=1, \ldots, L$. Let $a_{\ell}=0$ for all $\ell=L+1, \ldots, 2 L$. For the converse, consider a set $S$ such that $\sum_{\ell \in S} a_{\ell}=A$ and $|S|=L$. Let set $T=\{\ell: \ell \in S$ and $\ell \leq L\}$ and the result follows trivially.

Consider an instance of EqualCardinalitySubSETSum. Without loss of generality, assume that $0 \leq a_{1} \leq a_{2} \leq \ldots \leq a_{2 L}$. Moreover, we assume that $a_{2 L}>\sum_{\ell=1}^{2 L-1} a_{\ell}$ and $0<A<a_{2 L}$. These assumptions do not violate the NP-completeness of the problem. We define $a_{\ell}^{\prime}:=a_{\ell}+a_{2 L}$. For every integer $a_{\ell}$, we introduce a gadget (that is, $2 L$ gadgets in total). Every gadget $\ell$ consists of four items $i_{\ell, 1}, i_{\ell, 2}, i_{\ell, 3}, i_{\ell, 4}$ and a price vector $p_{\ell}$, which contains a price for each of the four items. Furthermore, there are $4+8 L$ bidders in every gadget $\ell$, one bidder is interested in bundle $\left\{i_{\ell, 1}\right\}$ with valuation $2 M-\frac{1}{2} a_{\ell}^{\prime}$, one bidder is interested in bundle $\left\{i_{\ell, 2}\right\}$ with valuation $a_{\ell}^{\prime}, 8 L$ bidders are interested in bundle $\left\{i_{\ell, 2}, i_{\ell, 3}\right\}$ with valuation $2 M+\left(\frac{1}{2}+\frac{1}{8 L}\right) a_{\ell}^{\prime}$, one bidder is interested in bundle $\left\{i_{\ell, 3}\right\}$ with valuation $2 M-\frac{1}{2} a_{\ell}^{\prime}$, and one bidder is interested in bundle $\left\{i_{\ell, 4}\right\}$ with valuation $M$, where $M$ is a sufficiently large integer. For gadget $2 L$, there is one additional bidder interested in bundle $\left\{i_{2 L, 1}, i_{2 L, 2}, i_{2 L, 3}, i_{2 L, 4}\right\}$ with valuation $5 M$. Finally, there is one big bidder interested in all items with valuation $10 M L+\frac{1}{8} a_{2 L}+\frac{1}{8 L} A$. Thus, the instance of the highway pricing problem has $2 L(4+8 L)+2$ bidders and $8 L$ items (see Figure 1 ).

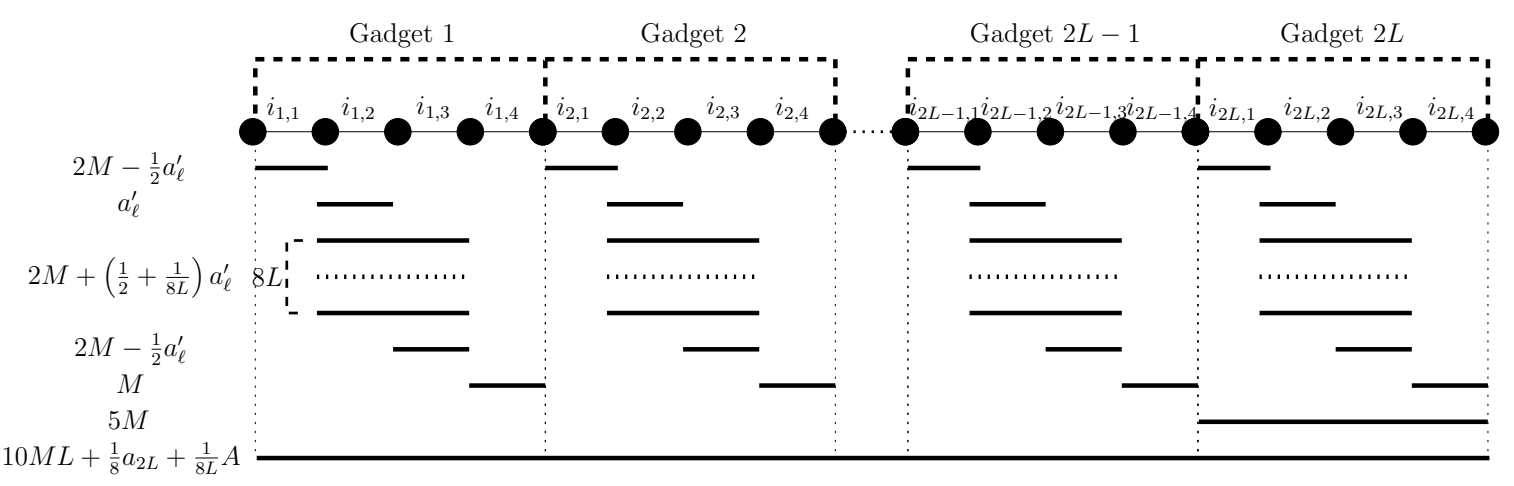

Fig. 1. Instance of the highway problem with global envy-freeness.

We claim that there exists a set $S \subseteq\{1, \ldots, 2 L\}$ such that $\sum_{\ell \in S} a_{\ell}=A$ and $|S|=L$ if and only if there is a feasible solution for the highway problem, fulfilling global envy-freeness, and with a total revenue of $(20+32 L) M L+5 M+4 L \sum_{\ell=1}^{2 L} a_{\ell}+\left(8 L^{2}+\frac{1}{8}\right) a_{2 L}+\frac{1}{8 L} A$. In the reduction we use two different price vectors, $p_{\ell}^{1}$ and $p_{\ell}^{2}$, selecting one of them for every gadget $\ell$. (We show in Lemma 3 that the solution fulfills global envy-freeness.) Price vector $p_{\ell}^{1}$ is defined as

$$
p_{\ell}^{1}=\left(2 M-\frac{1}{2} a_{\ell}^{\prime}, a_{\ell}^{\prime}, 2 M-\frac{1}{2} a_{\ell}^{\prime}, M\right),
$$

for which the total sum of the prices of the four items in gadget $\ell$ is equal to $5 M$ and the revenue from the bidders in this gadget (without big bidder) is $(5+16 L) M+4 L\left(a_{\ell}+a_{2 L}\right)$. 
The second price vector $p_{\ell}^{2}$ is defined as

$$
p_{\ell}^{2}=\left(2 M-\frac{1}{2} a_{\ell}^{\prime},\left(1+\frac{1}{8 L}\right) a_{\ell}^{\prime}, 2 M-\frac{1}{2} a_{\ell}^{\prime}, M\right),
$$

for which the total sum of the prices of the four items in gadget $\ell$ is equal to $5 M+\frac{1}{8 L} a_{\ell}^{\prime}$ and the revenue from the bidders in this gadget (without big bidder) is $(5+16 L) M+4 L\left(a_{\ell}+a_{2 L}\right)$. Note that by adding the condition $A<a_{2 L}$ for the EqualCardinalitySubsetSum problem, we assure that item $2 L \notin S$. By adding the bidder interested in bundle $\left\{i_{2 L, 1}, i_{2 L, 2}, i_{2 L, 3}, i_{2 L, 4}\right\}$ with valuation $5 M$, we assure that gadget $2 L$ is always priced $p_{2 L}^{1}$ as this yields a revenue in gadget $2 L$ of $(10+16 L) M+4 L\left(a_{\ell}+a_{2 L}\right)$.

$(\Rightarrow)$ Given a set $S \subseteq\{1, \ldots, 2 L\}$ such that $\sum_{\ell \in S} a_{\ell}=A$ and $|S|=L$. Let the price vector of gadget $\ell$ be $p_{\ell}^{1}$ if $\ell \notin S$, and $p_{\ell}^{2}$ if $\ell \in S$. Hence, the revenue of every gadget $\ell$ is $(5+16 L) M+4 L\left(a_{\ell}+a_{2 L}\right)$, independent of which price vector is used in gadget $\ell$, and there is an additional revenue in gadget $2 L$ of $5 M$. Given the pricing strategy for all items, the big bidder pays

$$
\sum_{\ell \in S}\left(5 M+\frac{1}{8 L}\left(a_{\ell}+a_{2 L}\right)\right)+\sum_{\ell \notin S}(5 M)=10 L M+\frac{1}{8} a_{2 L}+\frac{1}{8 L} A .
$$

Combining the contribution of the bidders in all gadgets and the big bidder yields a total revenue of

$$
\begin{aligned}
& \sum_{\ell=1}^{2 L}\left((5+16 L) M+4 L\left(a_{\ell}+a_{2 L}\right)\right)+5 M+10 L M+\frac{1}{8} a_{2 L}+\frac{1}{8 L} A \\
& =(20+32 L) M L+5 M+4 L \sum_{\ell=1}^{2 L} a_{\ell}+8 L^{2}+\frac{1}{8} a_{2 L}+\frac{1}{8 L} A .
\end{aligned}
$$

$(\Leftarrow)$ Given a feasible solution of the highway pricing problem with a total revenue of $(20+$ $32 L) M L+5 M+4 L \sum_{\ell=1}^{2 L} a_{\ell}+\left(8 L^{2}+\frac{1}{8}\right) a_{2 L}+\frac{1}{8 L} A$. The maximum revenue we can get from a bidder is her valuation. This would mean we receive $(5+16 L) M+(4 L+1) a_{\ell}^{\prime}$ per gadget $\ell$, and additionally $5 M$ for gadget $2 L$. However, there is no feasible pricing such that all bidders actually pay their valuation. The maximum we can get is $(5+16 L) M+4 L a_{\ell}^{\prime}$, by either using price vector $p_{\ell}^{1}$ or $p_{\ell}^{2}$ for gadgets $\ell=1, \ldots, 2 L-1$ and $(10+16 L) M+4 L a_{2 L}^{\prime}$ by pricing $p_{2 L}^{1}$ for gadget $2 L$. Given that the contribution in every gadget is $(5+16 L) M+4 L a_{\ell}^{\prime}$ with additionally $5 M$ for the last gadget, the big bidder should contribute at least

$$
\begin{aligned}
(20+32 L) M L+5 M+4 L \sum_{\ell=1}^{2 L} a_{\ell} & \left.+8 L^{2}+\frac{1}{8} a_{2 L}+\frac{1}{8 L} A-\sum_{\ell=1}^{2 L}(5+16 L) M+4 L a_{\ell}^{\prime}\right)-5 M \\
= & 10 M L+\frac{1}{8} a_{2 L}+\frac{1}{8 L} A .
\end{aligned}
$$

As this is the minimum amount the big bidder has to pay in an optimal solution, and it is equal to her valuation, she pays exactly the amount in (8). Let set $S=\left\{\ell: p_{\ell}=p_{\ell}^{2}\right\}$. Then, the actual payment can also be written as

$$
\sum_{\ell \in S}\left(5 M+\frac{1}{8 L}\left(a_{\ell}+a_{2 L}\right)\right)+\sum_{\ell \notin S}(5 M)=10 M L+\frac{1}{8 L}|S| a_{2 L}+\frac{1}{8 L} \sum_{\ell \in S} a_{\ell} .
$$

As the amounts in both, Equation (8) and (9), represent the actual contribution of the big bidder, they should be equal. That is, $\sum_{\ell \in S} a_{\ell}=(L-|S|) a_{2 L}+A$. We claim that 
$|S|=L$. To prove this, suppose it is not true. First, assume that $|S|<L$, then $\sum_{\ell \in S} a_{\ell}=$ $(L-|S|) a_{2 L}+A>\sum_{\ell=1}^{2 L-1} a_{\ell}+A$, which is not possible as $2 L \notin S$, so $|S| \geq L$. Now, assume $|S|>L$, then $\sum_{\ell \in S} a_{\ell}=(L-|S|) a_{2 L}+A<-a_{2 L}+A<0$, as $A<a_{2 L}$. This is also not possible as all integers $a_{\ell}$ are nonnegative. Therefore, we can conclude that $|S|=L$ and consequently, $A=\sum_{\ell \in S} a_{\ell}$.

Herewith we end the proof. What is left to show is that the above defined prices are globally envy-free.

Lemma 3. The pricing strategy used in the previous proof satisfies global envy-freeness.

Proof. In the previous proof, we assign either price vector $p_{\ell}^{1}$ or $p_{\ell}^{2}$ to gadget $\ell$. Moreover, we assume that $0 \leq a_{1} \leq a_{2} \leq \ldots \leq a_{2 L}$. We will determine a lower and upper bound on the price for a bundle of size $K$, where $K \leq n=8 L$. By the repetitiveness in the pricing strategy, the prices for bundles can be bounded as follows, depending on the bundle size $K$ (Table 1).

\begin{tabular}{|c|c|c|}
\hline Bundle size $K$ with: & Lower bound on price & Upper bound on price \\
\hline$K \equiv 0 \bmod 4$ & $5 M \frac{K}{4}+\frac{1}{2} a_{1}^{\prime}-\frac{1}{2} a_{2 L}^{\prime}$ & $5 M \frac{K}{4}-\frac{1}{2} a_{1}^{\prime}+\left(\frac{1}{2}+\frac{K}{4} \cdot \frac{1}{8 L}\right) a_{2 L}^{\prime}$ \\
$K \equiv 1 \bmod 4$ & $5 M \frac{K-1}{4}+a_{1}^{\prime}$ & $5 M \frac{K-1}{4}+2 M-\frac{1}{2} a_{1}^{\prime}+\frac{K-1}{4} \cdot \frac{1}{8 L} a_{2 L}^{\prime}$ \\
$K \equiv 2 \bmod 4$ & $5 M \frac{K-2}{4}+2 M+\frac{1}{2} a_{1}^{\prime}$ & $5 M \frac{K-2}{4}+3 M-\frac{1}{2} a_{1}^{\prime}+\frac{K-2}{4} \cdot \frac{1}{8 L} a_{2 L}^{\prime}$ \\
$K \equiv 3 \bmod 4$ & $5 M \frac{K-3}{4}+3 M+\frac{1}{2} a_{1}^{\prime}$ & $5 M \frac{K+1}{4}+\left(\frac{K-3}{4} \cdot \frac{1}{8 L}-1\right) a_{1}^{\prime}$ \\
\hline
\end{tabular}

Table 1. Smallest and largest bundle prices for bundles with size $K$.

One can easily calculate the difference between the lower bound of a bundle of size $K+1$ and the upper bound on a bundle of size $K, L B(K+1)-U B(K)$. As this difference is positive in all four cases, the global envy-freeness condition is satisfied. 